\title{
CHARACTERIZATION OF PESTICIDES AND PRACTICES OF COTTON PRODUCERS AND PESTICIDES SELLERS IN THE COTTON ZONE OF BALA'S HIPPOPOTAMUS POND BIOSPHERE OF BURKINA FASO
}

\section{Bazoma BAYILI $^{1,2 *}$, Richard OUEDRAOGO ${ }^{3,4}$, Sylvain ILBOUDO $^{4,5}$, Lamine POODA $^{2}$, Macaire BONKOUNGOU ${ }^{2}$, Jean Fidèle BATIONO ${ }^{6}$, Jean Bosco OUEDRAOGO ${ }^{1}$, Georges Anicet OUEDRAOGO ${ }^{2}$}

\author{
${ }^{1}$ Institute of Health Sciences Research, Regional office (IRSS-DRO/CNRST), Bobo Dioulasso, Burkina Faso \\ ${ }^{2}$ University of Nazi Boni (UNB), Bobo Dioulasso, Burkina Faso \\ ${ }^{3}$ Agricultural Institute of Environment and Agricultural Research, Regional office (INERA-DRO/ CNRST), Bobo Dioulasso, Burkina Faso \\ ${ }^{4}$ Joint International Research - Environment, Health and Society Unit (UMI 3189, ESS) CNRST/CNRS/UCAD/UGB/USTTB \\ ${ }^{5}$ Institute of Health Sciences Research, Bobo Dioulasso, Burkina Faso; (IRSS/CNRST), Ouagadougou, Burkina Faso \\ ${ }^{6}$ Muraz Center, Bobo-Dioulasso, Burkina Faso
}

Received - October 11, 2019; Revision - November 21, 2019; Accepted - December 22, 2019

Available Online - December 25, 2019

DOI: http://dx.doi.org/10.18006/2019.7(6).554.568

KEYWORDS
Cotton
Pesticides
Practices
Producers
Retailers
Bala's hippopotamus pond
biosphere
Burkina Faso

* Corresponding author

E-mail: bbazoma@yahoo.fr (Bazoma BAYILI)

Peer review under responsibility of Journal of Experimental Biology and Agricultural Sciences.

Production and Hosting by Horizon Publisher India [HPI] (http://www.horizonpublisherindia.in/).

All rights reserved.

\begin{abstract}
Chemical pesticides are systematically used against pests in conventional cotton growing. However, their use is known to be associated with health and environmental risks. Current study aimed to characterize pesticides and both crop producers and pesticide retailers practices. To this end, a survey and documentary research were carried out in the cotton zone around the biosphere of the Bala's hippopotamus pond, Burkina Faso. The results of study revealed that $87.5 \%$ of the pesticides retailers do not have agreement for pesticides selling. Further, more than $95 \%$ of the farmers wear their usual rural outfits to apply pesticides and $60.4 \%$ of them are not educated. The re-entry time into the treated fields was at most 24 hours for the majority of producers. Empty packaging is incinerated, abandoned in the wild or reused. About $50 \%$ of the producers experienced acute pesticide poisoning. The pesticide supply chain involves major distributors, retailers and pesticide users. Except LADABA, the 27 pesticides encountered at the producers, were identified at the retailers. Most of the used pesticides are mainly class III. In addition, $34.15 \%$ and $14.81 \%$ of pesticides from retailers and cotton producers are not
\end{abstract}

All the articles published by Journal of Experimental Biology and Agricultural Sciences are licensed under a Creative Commons Attribution-NonCommercial 4.0 International License Based on a work at www.jebas.org.

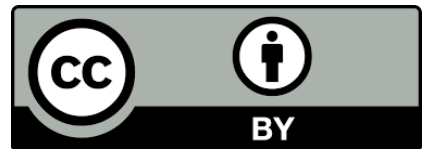


allowed for use by the Sahelian Pesticides Committee (CSP). Some of these pesticides were composed of banned active substances (endosulfan, atrazine and paraquat). Of the products used, imidacloprid, paraquat and spirotetramate are CMR active substances; deltamethrin and flubendiamide are endocrine disruptors. The regulation and continuous promotion of good practices for the use of pesticides approved from less toxic active substances should be favored in order to protect health and the environment.

\section{Introduction}

In Burkina Faso, cotton farming accounts for an average of $65 \%$ of households' cash income and contributes around $4 \%$ to national GDP and more than $28 \%$ to the country's agricultural GDP (MECV, 2011). However, cotton crop is confronted with the strong attacks of numerous pests including Helicoverpa armigera (Hübner) (Lepidoptera -Noctuidae), Diparopsis watersi (Rothchild) (Lepidoptera - Noctuidae), Earias spp. (Lepidoptera Noctuidae), greenfly Aphis gossypii (Glover) (Homoptera Aphididae) and defoliating caterpillars including Haritalodes derogata (Fabricius) (Lepidoptera, Pyralidae) (Achaleke \& Brévault, 2010; Badiane et al., 2015). The high pest pressure causes huge cotton crops losses which reached up to $70 \%$ of total yields (Traoré, 2008). To cope up with these losses farmers used pesticides for seed (insecticides and fungicides), soil (herbicides, nematicides and fungicides), and foliar treatments (insecticides, herbicides and fungicides) (Ouédraogo et al., 2011; FAO, 2015). In 2009 , the proportion of global pesticide sales for cotton was $6.2 \%$, compared to $29.7 \%$ for fruits and vegetables, $17 \%$ for cereals, $9.6 \%$ for soybean and $9.3 \%$ for maize (FAO, 2015). While in case of Burkina Faso, among the used pesticides, $90 \%$ of the pesticides are used in cotton production (PAN UK, 2018). Several pesticide formulations have been identified in Burkina Faso in the cotton zone. The main chemical groups of pesticides which used in cotton growing areas of Burkina Faso are Neonicotinoids, Pyrethroids and Organophosphorus (Badiane et al., 2015). As per survey conducted in 2015 , total 216 pesticide formulations was identified from the main cotton production area of the country, but $52 \%$ of these pesticides were not authorized by the current registration system (Conventionde Rotterdam, 2016).

As per the nature, physicochemical, biochemical and toxicological properties of pesticides, these can lead to major risks for human health (Saravi \& Shokrzadeh, 2011) and environment (Calvet et al., 2005). In addition, genetically modified cotton was introduced in Burkina Faso about ten years ago (2008-2016) (Sawadogo, 2016). Its abandonment marks the return to the intensive conventional culture whose multiplicity of treatments could induce a high level of human and environmental exposure.

Like most CILSS countries, the control and management of agricultural pesticides in Burkina Faso is governed by LAW $\mathrm{N}^{\circ}$
026-2017/AN (Assemblée Nationale, 2017). This law refers in particular to the strict conditions of distribution and sale of pesticides on the national market and advocates that employment in farming must be done according to good agricultural practices. Despite these legal management mechanisms, studies have shown that the distribution and supply chain of pesticides to producers remains partly fraudulent (Sory, 2011; Son et al., 2017). In addition, pesticide distribution practices would influence the conditions of their use by producers at the local level, and have an impact on human health and the environment. Proposed study is a part of global approach of risk analysis related to pesticides used in agriculture, based on an understanding of the practices of crop producers and sellers in the rural areas around the biosphere of the Bala's hippopotamus pond in Burkina Faso.

\section{Materials and Methods}

\subsection{Study framework and study population}

The study was conducted at the biosphere of the Bala's hippopotamus pond located in southwestern Burkina Faso about 60 $\mathrm{km}$ from Bobo-Dioulasso. Belem (2008) raised the risk of environmental degradation due to the high anthropization. Ecochemical risks associated with contamination of the pond by pesticides used on the watershed have also been suspected (Soleri, 2013). Indeed, the Satiri is an important cotton region of SOFITEX, involving the circulation and massive use of various pesticides. Thus, the pesticide dealers of Kadomba, Satiri, Bala and Tiarakô villages are attended in current study (Figure 1). All pesticide dealers encountered in the village markets were included in the study with their consent. While the cotton producers of the Tiarako, Sokourani and Bala villages, who have continuously produced cotton from last 3 seasons, including the 2018-2019 campaign, were sampled. The sample of producers was formed on the basis of 20 associations of 513 cotton producers, of which 139 producers were randomly selected. A minimum sample size of 82 producers was determined according to Rea \& Parker (2014) from the following formula:

$$
n=\frac{Z_{a}^{2} \times[p(1-p)] N}{Z_{a}^{2} \times[p(1-p)]+(N-1) M E_{p}^{2}}
$$

where $\mathrm{N}$ stands for population size, 『ME】_ $\mathrm{p}^{\wedge} 2$ for sampling error $(10 \%), \mathrm{p}$ for the true proportion set as 0.5 and $\mathrm{Za}$ is equal to 1.96 for $95 \%$ confidence level. 


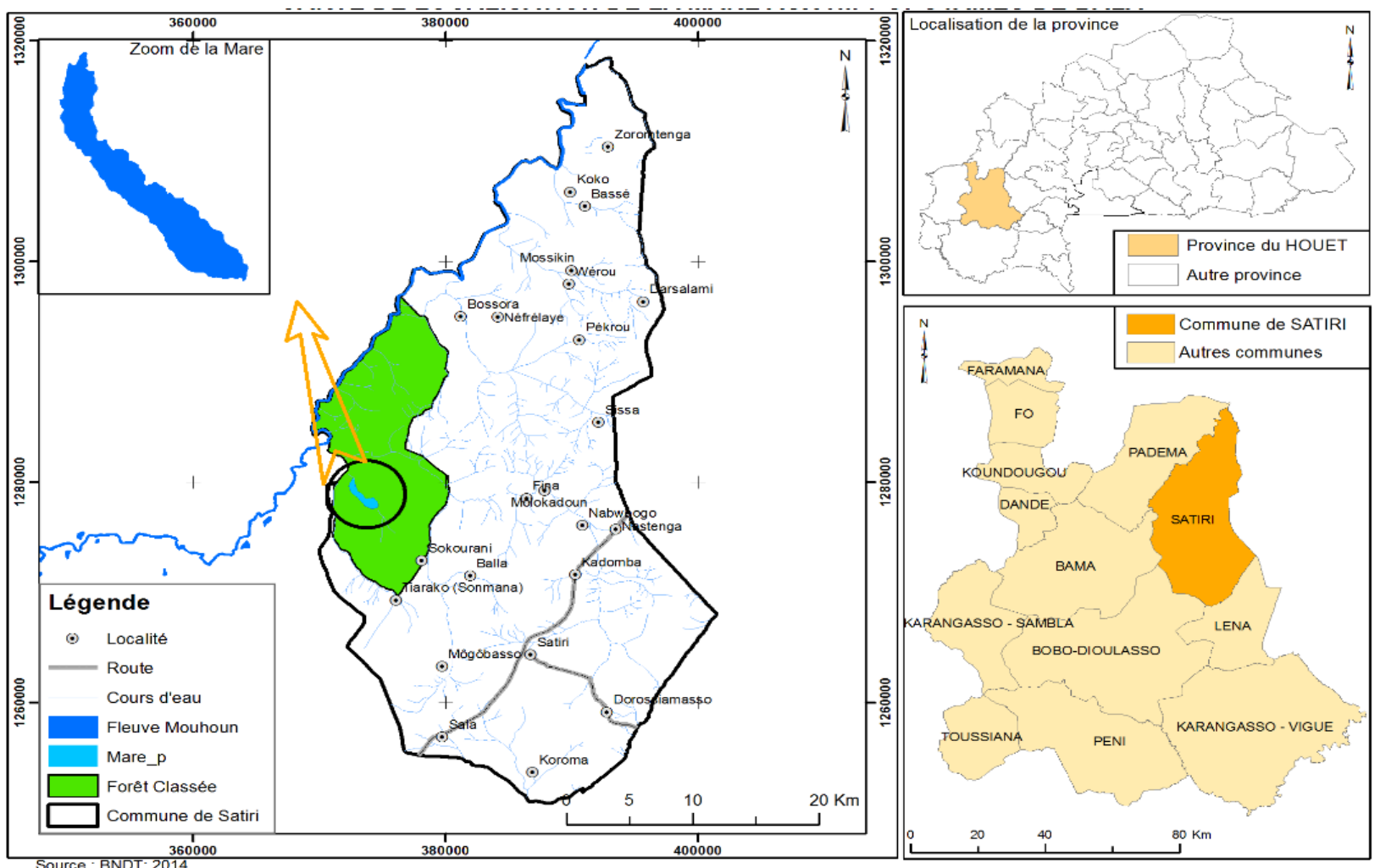

Figure 1 Geographical location of the study site

\subsection{Surveys}

Cross-sectional surveys based on personal interviews were conducted from 1 June to 15 September 2018 with pesticides retailers while in case of cotton producers survey was conducted from 25 September to 10 October 2018. An individual semistructured questionnaire was designed and administered in the form of a semi-structured interview to 8 retailers and sampled producers.

For retailers, field trips have been planned to list all products used during the campaign. The information collected from the socioprofessional characteristics of the cotton producers and pesticides retailers, the commercial pesticide specialties, the marketing / use mode, the pesticide supply system, etc. Also, the questionnaire administered to cotton producers included a section on reported cases of poisoning.

\subsection{Characterization of pesticides}

The characterization of pesticides was made for commercial specialties and active substances by documentary research. Thus, the positive global list of pesticides approved by the Sahelian Committee for Pesticides (CSP) was used to know in particular the registration status and the use of pesticides. WHO Recommended Classification of Pesticides by Hazard (WHO, 2010) used to determine "WHO toxicity class" of pesticides, is given in table 1. Carcinogenic, mutagenic and reprotoxic (CMR) and endocrine disruptor status of the pesticide active substances inventoried were determined from the Pesticide / Biopesticide Properties Database (PPDB / BPDB).

Table 1 WHO Recommended Classification of Pesticides by Hazard

\begin{tabular}{|c|c|c|c|}
\hline \multirow{2}{*}{$\begin{array}{l}\text { WHO } \\
\text { Class }\end{array}$} & \multirow[t]{2}{*}{ Category } & \multicolumn{2}{|c|}{$\begin{array}{l}\mathrm{LD}_{50} \text { for the rat }(\mathrm{mg} / \mathrm{kg} \\
\text { body weight) }\end{array}$} \\
\hline & & Oral & Dermal \\
\hline Ia & Extremely hazardous & $<5$ & $<50$ \\
\hline $\mathrm{Ib}$ & Highly hazardous & $5-50$ & $50-200$ \\
\hline II & Moderately hazardous & $50-2000$ & $200-2000$ \\
\hline III & Slightly hazardous & Over 2000 & Over 2000 \\
\hline $\mathrm{U}$ & $\begin{array}{l}\text { Unlikely to present acute } \\
\text { hazard }\end{array}$ & $5000 \mathrm{c}$ & iigher \\
\hline
\end{tabular}

Table information source: WHO (2010)

\subsection{Data analysis}

The pesticide characteristic data and the distributor survey data were manually processed before being entered and analyzed with Excel 2013 software. Further producers' practices were analyzed with SPSS v20 software. Descriptive statistics focused on simple 
frequencies and averages. Producer practices were analyzed by comparison with good agricultural and phytosanitary practices published in the International Code of Conduct for the Distribution and Use of Pesticides (FAO/OMS, 2014).

\section{Results}

\subsection{Sociodemographic Characteristics}

The pesticide retailers encountered ( 8 in total) were male with an average of 6 years in the sale of pesticides. Among the studied retailers, only one had an approval for pesticides sale. In addition, $87.5 \%$ of retailers had no knowledge of the CSP and $75 \%$ did not have any updated list of products approved by the CSP. Further, only one had safety data sheets in his store and a first aid kit containing medication. In case of producer, all crop producers were male with an average age of 39 years old. Among these, the youngest one is 18 years old and the oldest one is 60 years old. In case of educational status, $60.4 \%$ of the sellers were illiterate, $23 \%$ had education only up to primary school level while $16.5 \%$ had secondary school level education. Almost half of the producers (45.3\%) had more than 20 years of pesticide used experience (Figure 2).

\subsection{Pesticide supply sources}

Pesticide distributors generally obtained their supplies from whole sellers and phytopharmaceutical companies (such as SOLEVO, Saphyto, BaryAgrochem, Prophyma, Senefura, Af-chem, GNESSIN Establishment) in Bobo-Dioulasso. Cotton producers obtain almost all pesticides from retailers in local markets and insecticides from SOFITEX (Table 2).

\subsection{Cotton producers and pesticides retailers training level}

Thirty seven percent $(37.5 \%)$ of the retailers received training on the safe management of pesticides, provided by the International Fertilizer Development Center (IFDC), Plant Protection and Packaging Direction (DPVC), the Agricultural Inputs Wholesalers and Retailers Association of Burkina Faso (AGRODIA), African Society of Phytosanitary Products and Insecticides (SAPHYTO) and SOLEVO. As for cotton producers, $69.6 \%$ received training on a various set of themes related to the pesticides use (Figure 3 ).

\subsection{Sale of pesticides}

Among the studied seller respondents, 50\% retailers are associated with the selling of food and other consumer products such as corn, coffee, rice along with the pesticides in the same shop. Two surveyed retailers said producers returned to them purchased pesticides. All retailers gave on choice information regarding the risks and use of pesticides to the producers. No seller was selling Personal Protective Equipment (PPE).

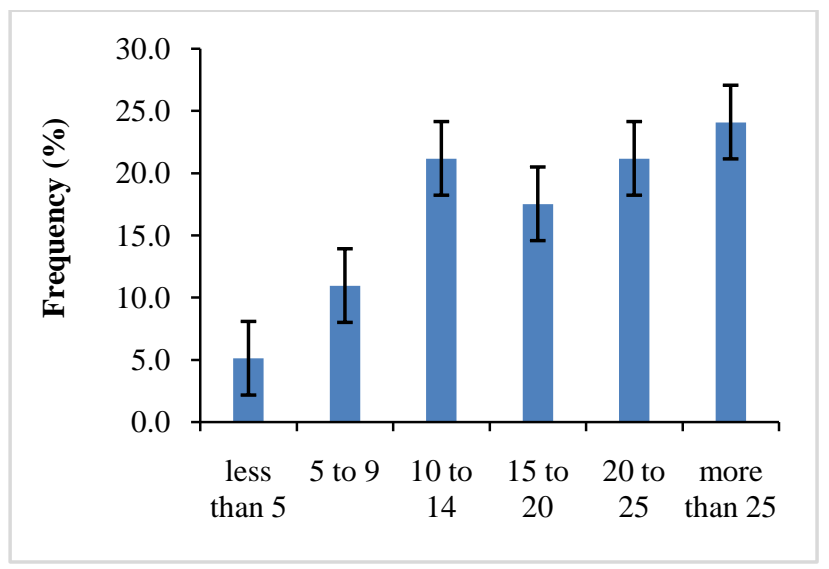

Figure 2 Producers' experience in cotton production (The bars corresponded to the standard error which represents mean $\pm \mathrm{SE}$ )

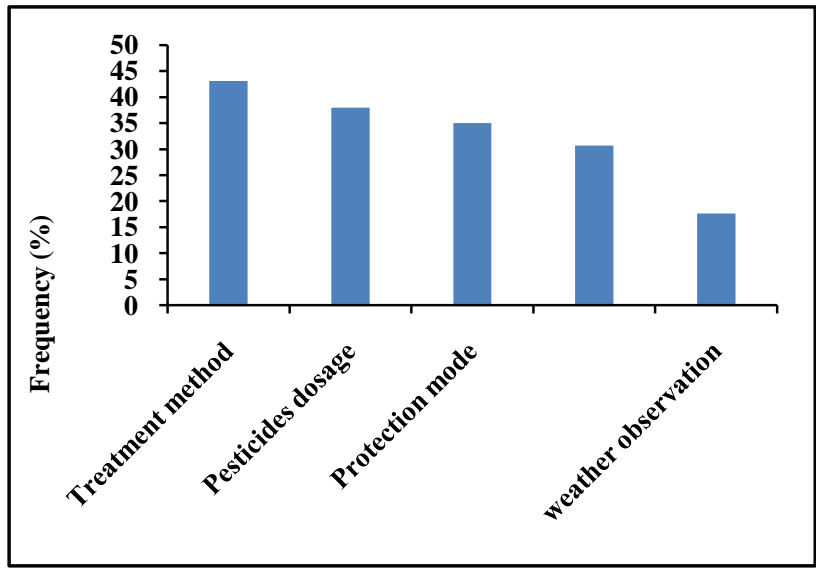

Figure 3 Various training taken by crop producers' during pesticides handling

Table 2 pesticides supply sources of cotton producers

\begin{tabular}{|cccccccccccccccccc}
\hline $\begin{array}{c}\text { Sources } \\
\text { Types }\end{array}$ & I & H & F & I & H & F & I & H & F & I & H & F & I & H & F \\
\hline Effective & 139 & 65 & 12 & 0 & 118 & 0 & 0 & 12 & 0 & 0 & 3 & 0 & 0 & 2 & 0 \\
\hline Frequency (\%) & 100 & 46.76 & 8.63 & 0 & 84.89 & 0 & 0 & 8.63 & 0 & 0 & 2.16 & 0 & 0 & 1.44 & 0 \\
\hline
\end{tabular}

$I$ : insecticide $; H$ : herbicide $; F$ : fungicide 


\subsection{Use and management modes of pesticides}

The choice of pesticides to be used is made by $63.3 \%$ of the cotton producers themselves but some time it depends on the choice of technical services providers $(59.4 \%)$ to the producers (Figure 4). Producers are rarely on the use of conventional PPE in pesticide treatment operations (Figure 5). Almost all producers (95.7\%) used their usual field clothing during treatment sessions and rarely $(2.2 \%)$ they use suitable suits made for pesticides application (Figure 6). Also, 27.5\% of the producers used combination of certain pesticides to treat crops.

After the use of pesticides, several methods of used packaging materials management are adopted by the producers (Figure 7). They went through incineration (43.9\% of producers), desertion in nature $(42.8 \%)$ and even in domestic use $(5.1 \%)$ (Figure 8). Several re-entry times are observed by producers, with approximately $70 \%$ waiting no more than 24 hours (Figure 9). Most producers

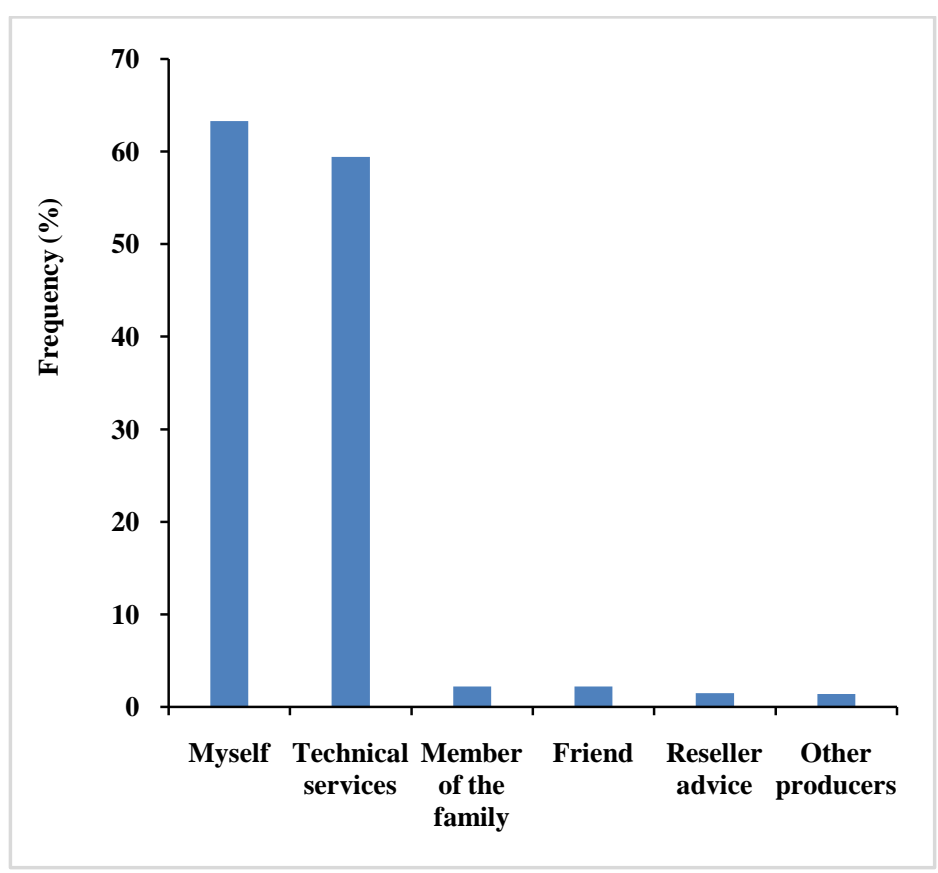

Figure 4 Various criteria for pesticides choosing by producers
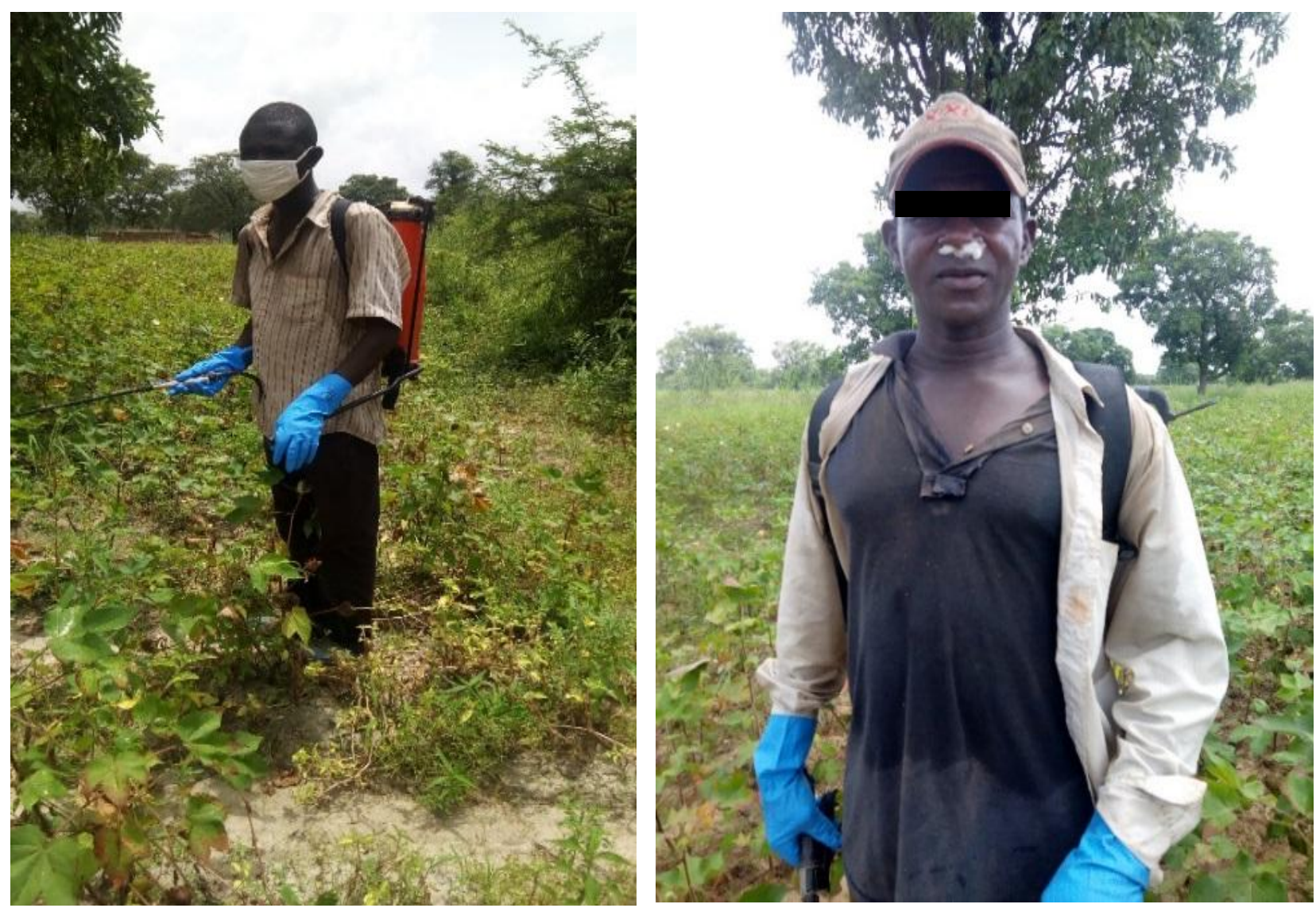

Figure 5 Precautions taken by crop producers during pesticides handlings

Journal of Experimental Biology and Agricultural Sciences http://www.jebas.org 


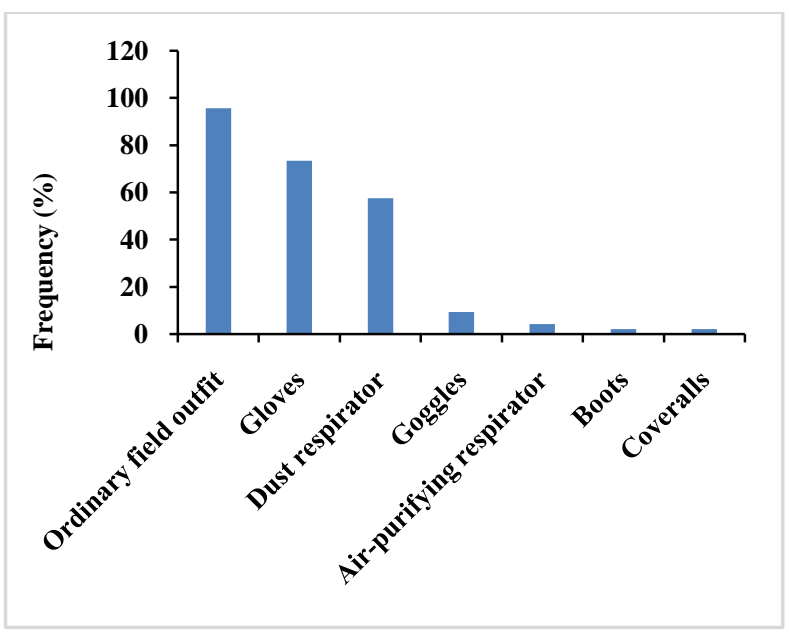

Figure 6 Different protection equipment used by crop producers during pesticides handlings

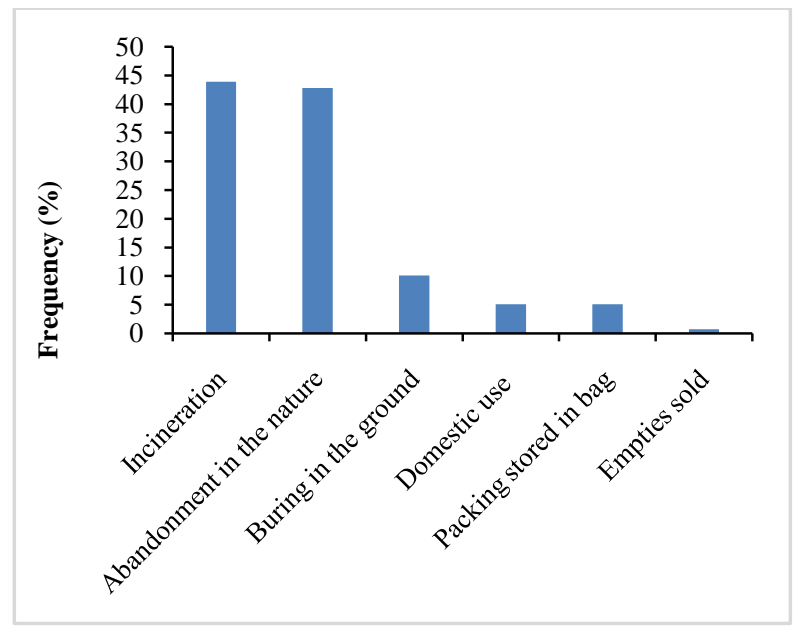

Figure 7 Management mode of empty packaging after treatment
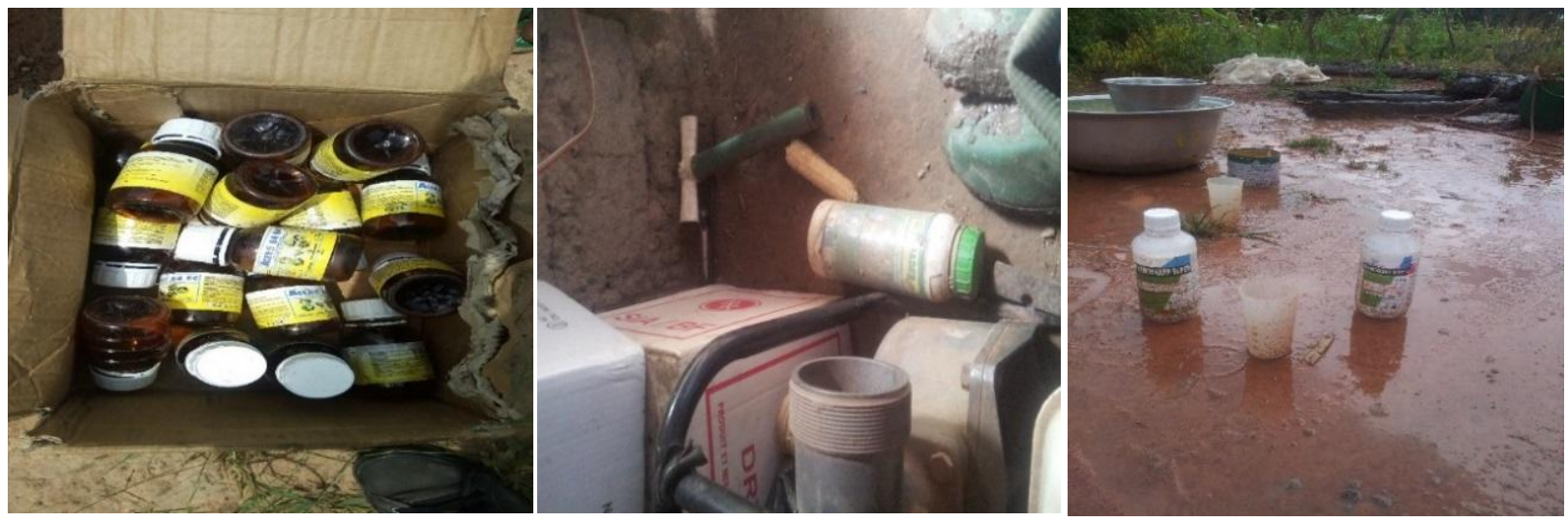

Figure 8 (a) Empty packaging for incineration; (b) Empty packaging in a dwelling; (c) Abandoned packaging at the edge of a well

have water points (well, ravine, river, ponds) in their field or nearby, and they use this water for different purposes. Thus, $65.1 \%$ use them for drinking, $33.6 \%$ for pesticide processing activities, $29.9 \%$ for domestic use and $20.4 \%$ for watering domestic animals (Figure 10). Most of the producer used these water sources for prepared stock solution of pesticides and there is a great chance of spreading contamination during operation or handling these pesticides.

\subsection{Cases of poisoning reported by producers}

About half of the producers (49.64\%) affirmed that they have felt signs of poisoning a few moments after pesticide application operations (Figure 11). During survey most common symptoms of pesticide poisoning faced by producers are itchiness (25.5\%), eye burns (18.0\%), dizziness $(5.8 \%)$, various irritations $(5.0 \%)$, skin irritations and headaches $(3.6 \%)$, fever (2.2\%) and stomach pain $(0.7 \%)$.

\subsection{Recommendations and suggestions from producers}

Producers made recommendations / suggestions for their capacity building $(32.1 \%)$, the reduction of the number of pesticides treatment $(26.4 \%)$, accessibility to medical visits after treatment (24.8\%), the availability and accessibility of PPE (21.7\%), the reduction of the pesticide prices $(13.3 \%)$ and the control of the pesticides sale (3.1\%) (Figure 12).

\subsection{Main identified pesticides}

Eighty-three (83) commercial specialties based on 40 active substances were identified at retailers'(levels) during the campaign and 27 commercial specialties consisting of 20 active substances were identified at producers', among these 26 are identical to those found at retailers' levels. Of the identified pesticides from producers in particular, $77.78 \%$ were herbicides, $18.52 \%$ insecticides and 


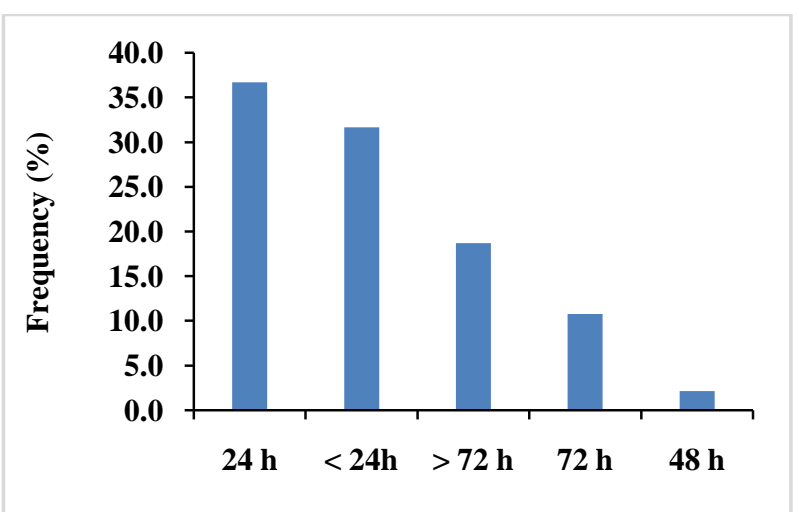

Figure 9 Reentry times in the field after treatment

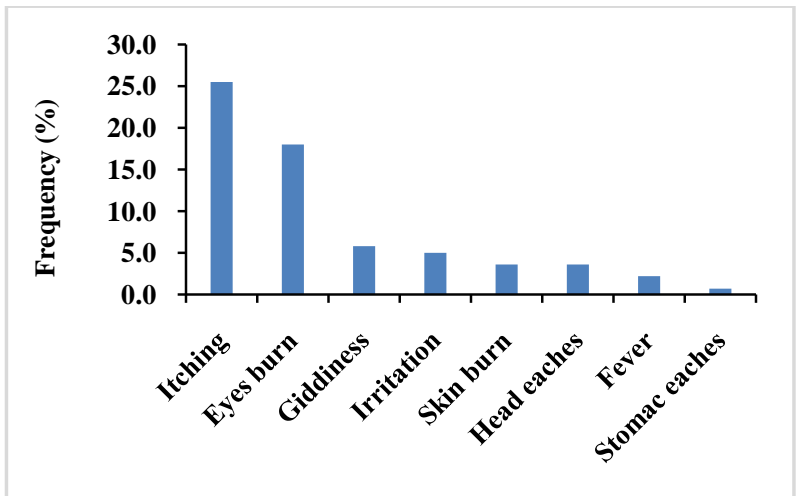

Figure 11 Different signs of intoxication felt by crop producers during pesticide application

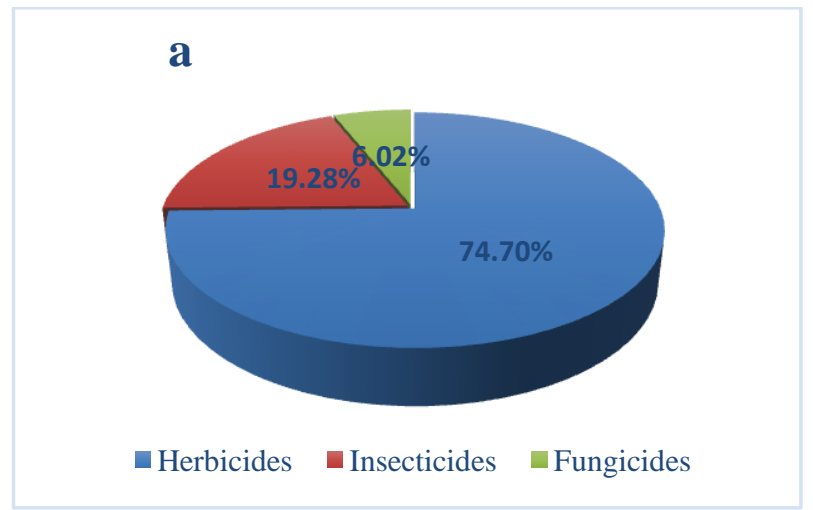

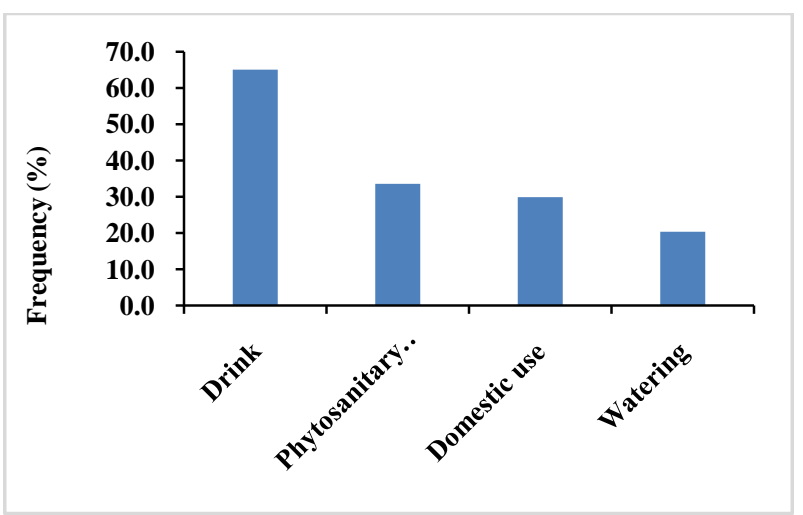

Figure 10 Different uses of the existing water point in the field

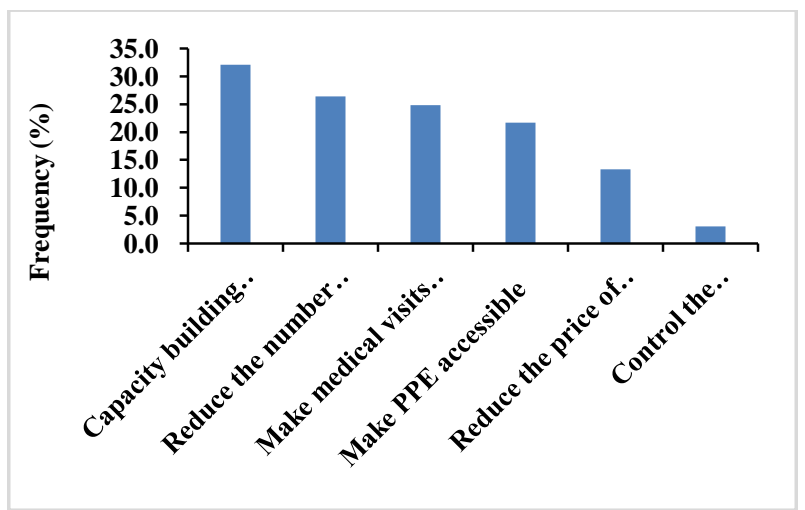

Figure 12 Recommendations related pesticides handling given by crop producers'

Figure 13 Proportion of each type of pesticide (a) in markets and (b) from producers

$3.70 \%$ fungicides (Figures 13a and 13b). The only product exclusively found among producers is KALACH. Glyphosate, nicosulfuron and paraquat are the active ingredient in most commonly used herbicide found at distributors', while at producers' these are glyphosate, diuron, haloxyfop-p-methyl, nicosulfuron and paraquat. Unregistered products accounted for $34.15 \%$ of the pesticides found in pesticides retailers (Figure 13a), including substances such as atrazine, endosulfan and paraquat.
For producers, these products accounted for $14.81 \%$ and also were composed of paraquat (Figure 13b).THUNDER, an insecticide which has been identified in retail stores, is an approved binary product containing Class Ib beta-cyfluthrin. All of the pesticides identified from producers and their characteristics are recorded in Table 3. Pesticides incriminated by producers as causing poisoning are mainly, PYRINEX QUICK 424EC (deltamethrin and chlorpyrifos-ethyl) (25.9\%), TIHAN 175 O-TEQ (Spirotetremate 
Table 3 List of pesticides encountered from retailers and producers

\begin{tabular}{|c|c|c|c|c|c|c|}
\hline Commercial formulation & $\begin{array}{l}\text { Type of } \\
\text { pesticide }\end{array}$ & $\begin{array}{l}\text { WHO } \\
\text { class }\end{array}$ & $\begin{array}{l}\text { CSP } \\
\text { registration } \\
\text { status }\end{array}$ & $\begin{array}{c}\text { Active substances } \\
\text { (a.s.) }\end{array}$ & Group of substances & $\begin{array}{l}\text { WHO } \\
\text { class of } \\
\text { a.s. }\end{array}$ \\
\hline \multirow{2}{*}{ *ACERO $84 \mathrm{EC}^{3}$} & \multirow{2}{*}{ I } & \multirow{2}{*}{ II } & \multirow{2}{*}{ Yes } & $\begin{array}{l}\text { Isoclast } \\
\text { (sulfoxaflor) }\end{array}$ & Sulfoximine & III \\
\hline & & & & Lambda-cyhalothrin & Pyrethroid & II \\
\hline *ACTION 80 DF & $\mathrm{H}$ & III & Yes & Diuron & Phenylamide & III \\
\hline *ADWUMA WURA & $\mathrm{H}$ & $\mathrm{U}$ & No & Glyphosate & Phosphonoglycine & III \\
\hline ALLIGATOR & $\mathrm{H}$ & III & Yes & Pendimethalin & Dinitroaniline & II \\
\hline ATRAHERB WP & $\mathrm{H}$ & II & No & Atrazine & Triazine & III \\
\hline ATRAZILA $80 \mathrm{WP}$ & $\mathrm{H}$ & II & No & Atrazine & Triazine & III \\
\hline ATRAZILA 500 SC & $\mathrm{H}$ & II & No & Atrazine & Triazine & III \\
\hline \multirow{2}{*}{ ATTAKAN C 344 SC } & \multirow{2}{*}{ I } & \multirow{2}{*}{ II } & \multirow{2}{*}{ Yes } & Imidacloprid & Neonicotinoid & II \\
\hline & & & & Cypermethrin & Pyrethroid & II \\
\hline *AVAUNT $150 \mathrm{EC}^{1}$ & I & III & Yes & Indoxacarb & Oxadiazine & II \\
\hline \multirow{2}{*}{ BACCARA } & \multirow{2}{*}{$\mathrm{H}$} & \multirow{2}{*}{ II } & \multirow{2}{*}{ Yes } & 2,4-D & Aryloxyacide & II \\
\hline & & & & Propanyl & Anilide & II \\
\hline $\begin{array}{l}\text { BENAXONE SUPER } 200 \\
\text { SL }\end{array}$ & $\mathrm{H}$ & II & No & Paraquat & Bipyridylium & II \\
\hline $\begin{array}{l}\text { BENAXONE SUPER } 276 \\
\text { SL }\end{array}$ & $\mathrm{H}$ & II & No & Paraquat & Bipyridylium & II \\
\hline BINBEFLA PLUS 720 SL & $\mathrm{H}$ & III & Yes & 2,4-D amine salt & Aryloxyacide & II \\
\hline BONNICO & $\mathrm{H}$ & III & No & Nicosulfuron & sulfonylurea & $\mathrm{U}$ \\
\hline BONQUAT & $\mathrm{H}$ & II & No & Paraquat & Bipyridylium & II \\
\hline \multirow{2}{*}{ CAIMAN ROUGE } & \multirow{2}{*}{ I } & \multirow{2}{*}{ II } & \multirow{2}{*}{ No } & Endosulfan & Organochlorine & II \\
\hline & & & & Thiram & Carbamate & II \\
\hline \multirow{2}{*}{ CAIMAN ROUGE P } & \multirow{2}{*}{ I } & \multirow{2}{*}{ II } & \multirow{2}{*}{ Yes } & Permethrin & Pyrethroid & II \\
\hline & & & & Thirame & Carbamate & II \\
\hline \multirow{2}{*}{ CALTHIO E } & \multirow{2}{*}{$\mathrm{F}$} & \multirow{2}{*}{ II } & \multirow{2}{*}{ No } & Endosulfan & Organochlorine & II \\
\hline & & & & thiram & Carbamate & II \\
\hline \multirow{3}{*}{ CALTHIO MIX 485 WS } & \multirow{3}{*}{$\mathrm{F}$} & \multirow{3}{*}{ II } & \multirow{3}{*}{ Yes } & Imidacloprid & Neonicotinoid & II \\
\hline & & & & Thiram & Carbamate & II \\
\hline & & & & Metalaxyl & Phenylamide & II \\
\hline CORAGEN 20 SC & $\mathrm{H}$ & $\mathrm{U}$ & Yes & chlorantraniliprole & Diamide anthranilique & $\mathrm{U}$ \\
\hline CORIGNFNA 500 FC & $\mathrm{H}$ & UI & Yes & Terbutryn & Triazine & III \\
\hline CORIUNENA SOU EC & $\mathrm{H}$ & III & Yes & metolachlor & Chloroacetamide & III \\
\hline DEKADE PLUS 720 SL & $\mathrm{H}$ & III & Yes & 2,4-D amine salt & Aryloxyacide & II \\
\hline *DIGA-FAGALAN 360 SL & $\mathrm{H}$ & III & Yes & glyphosate & Phosphonoglycin & III \\
\hline *DIURALM 80 WG & $\mathrm{H}$ & III & Yes & Diuron & Phenylamide & III \\
\hline DIURON PLUS & $\mathrm{H}$ & III & No & Diuron & Phenylamide & III \\
\hline
\end{tabular}




\begin{tabular}{|c|c|c|c|c|c|c|}
\hline Commercial formulation & $\begin{array}{l}\text { Type of } \\
\text { pesticide }\end{array}$ & $\begin{array}{l}\text { WHO } \\
\text { class }\end{array}$ & $\begin{array}{l}\text { CSP } \\
\text { registration } \\
\text { status }\end{array}$ & $\begin{array}{l}\text { Active substances } \\
\text { (a.s.) }\end{array}$ & Group of substances & $\begin{array}{l}\text { WHO } \\
\text { class of } \\
\text { a.s. }\end{array}$ \\
\hline \multirow{2}{*}{ *EMAPYR ${ }^{3}$} & \multirow{2}{*}{ I } & \multirow{2}{*}{ III } & \multirow{2}{*}{ Yes } & $\begin{array}{l}\text { Emamectin } \\
\text { benzoate }\end{array}$ & Derivedfrommicroorganisms & II \\
\hline & & & & Pyriproxyfen & Pyridine derived & $\mathrm{U}$ \\
\hline *GALLANT SUPER & $\mathrm{H}$ & III & Yes & $\begin{array}{l}\text { Haloxyfop-p- } \\
\text { methyl }\end{array}$ & Aryloxyphenoxypropionate & II \\
\hline \multirow{2}{*}{ GENERAL } & \multirow{2}{*}{$\mathrm{F}$} & \multirow{2}{*}{ II } & \multirow{2}{*}{ No } & Endosulfan & Organochlorine & II \\
\hline & & & & Thiram & Carbamate & II \\
\hline *GLYCEL 410 SL & $\mathrm{H}$ & II & Yes & Glyphosate & Phosphonoglycin & III \\
\hline GLYCOT 700 SG & $\mathrm{H}$ & III & No & $\begin{array}{l}\text { Glyphosate, } \\
\text { isopropylaminesalt }\end{array}$ & Phosphonoglycin & III \\
\hline *GLYPHADER 75 SG & $\mathrm{H}$ & III & Yes & Glyphosate & Phosphonoglycin & III \\
\hline *GLYPHADER 360 SL & $\mathrm{H}$ & $\mathrm{U}$ & Yes & Glyphosate & Phosphonoglycin & III \\
\hline GLYPHADER 68 SG & $\mathrm{H}$ & III & No & Glyphosate & Phosphonoglycin & III \\
\hline GLYPHALM 360 SL & $\mathrm{H}$ & III & Yes & Glyphosate & Phosphonoglycin & III \\
\hline *GLYPHE & $\mathrm{H}$ & $\mathrm{U}$ & Yes & Glyphosate & Phosphonoglycin & III \\
\hline *GLYPHOBAR 480 SL & $\mathrm{H}$ & III & Yes & $\begin{array}{l}\text { Glyphosate, } \\
\text { isopropylaminesalt }\end{array}$ & Phosphonoglycin & III \\
\hline GLYSTAR 360 SL & $\mathrm{H}$ & III & Yes & Glyphosate & Phosphonoglycin & III \\
\hline *GRAMOSHAP SUPER & $\mathrm{H}$ & II & No & Paraquatdichloride & $\begin{array}{l}\text { Quaternary ammonium } \\
\text { compound }\end{array}$ & II \\
\hline HALODAF 108 EC & $\mathrm{H}$ & III & Yes & $\begin{array}{l}\text { Haloxyfop-p- } \\
\text { methyl }\end{array}$ & Aryloxyphenoxypropionate & II \\
\hline *HALONET 104 EC & $\mathrm{H}$ & III & Yes & $\begin{array}{l}\text { Haloxyfop-p- } \\
\text { methyl }\end{array}$ & Aryloxyphenoxypropionate & II \\
\hline HERBEXTRA 720 SL & $\mathrm{H}$ & II & Yes & 2,4-D amine salt & Aryloxyacide & II \\
\hline \multirow{2}{*}{ HERBIMAIS $240 \mathrm{OF}$} & \multirow{2}{*}{$\mathrm{H}$} & \multirow{2}{*}{ III } & \multirow{2}{*}{ Yes } & Dicamba & Benzoicacid & II \\
\hline & & & & Nicosulfuron & Sulfonylurea & $\mathrm{U}$ \\
\hline IKOKADIGNE & $\mathrm{H}$ & II & Yes & $\begin{array}{l}\text { Haloxyfop-p- } \\
\text { methyl }\end{array}$ & Aryloxyphenoxypropionate & II \\
\hline \multirow{2}{*}{ *IMIDALM-T $450 \mathrm{WS}$} & \multirow{2}{*}{$\mathrm{F}$} & \multirow{2}{*}{ II } & \multirow{2}{*}{ Yes } & Imidacloprid & Neonicotinoid & II \\
\hline & & & & Thiram & Carbamate & II \\
\hline INDOXAN & I & III & Yes & Indoxacarb & Oxadiazine & II \\
\hline KABABIN $40 \mathrm{SC}$ & $\mathrm{H}$ & III & Yes & Nicosulfuron & sulfonylurea & $\mathrm{U}$ \\
\hline **KALACH $360 \mathrm{SL}$ & $\mathrm{H}$ & III & Yes & Glyphosate & Phosphonoglycin & III \\
\hline *KILLER $450 \mathrm{SL}$ & $\mathrm{H}$ & $\mathrm{U}$ & Yes & Glyphosate & Phosphonoglycin & III \\
\hline *LADABA & $\mathrm{H}$ & $\mathrm{U}$ & No & Glyphosate & Phosphonoglycin & III \\
\hline *LAMACHETTE 360 SL & $\mathrm{H}$ & III & Yes & Glyphosate & Phosphonoglycin & III \\
\hline LAMBDA POWER & I & II & No & $\begin{array}{l}\text { Lambda- } \\
\text { Cyhalothrin }\end{array}$ & Pyrethroid & II \\
\hline $\begin{array}{l}\text { LAMBDRAF SUPER } 2,5 \\
\text { EC }\end{array}$ & I & II & Yes & Lambda-cyhalothrin & Pyrethroid & II \\
\hline \multirow[b]{2}{*}{ LAMBDAQUEST 62 EC } & \multirow{2}{*}{ I } & \multirow[b]{2}{*}{ II } & \multirow[b]{2}{*}{ No } & acetamiprid & Neonicotinoid & II \\
\hline & & & & Lambda-cyhalothrin & Pyrethroid & II \\
\hline
\end{tabular}




\begin{tabular}{|c|c|c|c|c|c|c|}
\hline Commercial formulation & $\begin{array}{c}\text { Type of } \\
\text { pesticide }\end{array}$ & $\begin{array}{l}\text { WHO } \\
\text { class }\end{array}$ & $\begin{array}{l}\text { CSP } \\
\text { registration } \\
\text { status }\end{array}$ & $\begin{array}{l}\text { Active substances } \\
\text { (a.s.) }\end{array}$ & Group of substances & $\begin{array}{l}\text { WHO } \\
\text { class of } \\
\text { a.s. }\end{array}$ \\
\hline \multirow{2}{*}{ *LIBERATOR $500 \mathrm{SC}$} & \multirow{2}{*}{$\mathrm{H}$} & \multirow{2}{*}{ III } & \multirow{2}{*}{ Yes } & Diflufenican & Carboxamide & III \\
\hline & & & & Flufenacet & Oxyacetamide & II \\
\hline MAIA 75 WG & $\mathrm{H}$ & III & Yes & Nicosulfuron & Sulfonylurea & $\mathrm{U}$ \\
\hline MALIK 108 EC & $\mathrm{H}$ & III & Yes & Haloxyfop-p-methyl & Aryloxyphenoxypropionate & II \\
\hline \multirow{2}{*}{ MEPRODAF 510 EC } & \multirow{2}{*}{$\mathrm{H}$} & \multirow{2}{*}{ III } & \multirow{2}{*}{ Yes } & metolachlor & Chloroacetamide & III \\
\hline & & & & Promethryn & Triazine & III \\
\hline \multirow{2}{*}{ MOMTAZ 45 WS } & \multirow{2}{*}{$\mathrm{F}$} & \multirow{2}{*}{ III } & \multirow{2}{*}{ Yes } & Imidacloprid & Neonicotinoid & II \\
\hline & & & & Thiram & Carbamate & II \\
\hline NICOBAK & $\mathrm{H}$ & $\mathrm{U}$ & No & Nicosulfuron & Sulfonylurea & $\mathrm{U}$ \\
\hline NICODAF 40 SC & $\mathrm{H}$ & III & Yes & Nicosulfuron & Sulfonylurea & $\mathrm{U}$ \\
\hline NICOLABAN 40 SC & $\mathrm{H}$ & III & Yes & Nicosulfuron & Sulfonylurea & $\mathrm{U}$ \\
\hline *NICOMAIS 40 SC & $\mathrm{H}$ & III & Yes & Nicosulfuron & Sulfonylurea & $\mathrm{U}$ \\
\hline NICONET $40 \mathrm{SC}$ & $\mathrm{H}$ & III & Yes & Nicosulfuron & Sulfonylurea & $\mathrm{U}$ \\
\hline NICOPAR SUPER & $\mathrm{H}$ & $\mathrm{U}$ & No & Nicosulfuron & Sulfonylurea & $\mathrm{U}$ \\
\hline NICOPAT SUPER 40 OD & $\mathrm{H}$ & II & No & Nicosulfuron & Sulfonylurea & $\mathrm{U}$ \\
\hline NICOPLUS 40 OD & $\mathrm{H}$ & III & No & Nicosulfuron & Sulfonylurea & $\mathrm{U}$ \\
\hline ONYX $360 \mathrm{EC}$ & $\mathrm{H}$ & III & No & S-metolachlor & Chloroacetamide & III \\
\hline PINGRAZONE & $\mathrm{H}$ & $\mathrm{U}$ & No & Paraquatchloride & $\begin{array}{l}\text { Quaternary ammonium } \\
\text { compound }\end{array}$ & II \\
\hline \multirow{2}{*}{ POLYTRINE C 186 EC } & \multirow{2}{*}{ I } & \multirow{2}{*}{ II } & \multirow{2}{*}{ No } & Cypermethrin & Pyrethroid & II \\
\hline & & & & Profenofos & Organophosphate & II \\
\hline *POWER & $\mathrm{H}$ & III & Yes & Diuron & & III \\
\hline \multirow{2}{*}{$\begin{array}{l}\text { *PYRINEXQUICK } 424 \\
\text { EC }^{2}\end{array}$} & \multirow{2}{*}{ I } & \multirow{2}{*}{ II } & \multirow{2}{*}{ Yes } & Deltamethrin & Pyrethroid & II \\
\hline & & & & Chlorpyrifos-ethyl & Organophosphate & III \\
\hline *ROUNDUP 360 XL & $\mathrm{H}$ & III & Yes & Glyphosate & Phosphonoglycin & III \\
\hline RUBIS $100 \mathrm{SC}$ & $\mathrm{H}$ & III & Yes & Bispyribac-sodium & $\begin{array}{l}\text { Pyrimidinylcarboxy } \\
\text { compound }\end{array}$ & III \\
\hline SAMORY & $\mathrm{H}$ & III & Yes & BensulfuronMethyl & Sulfonylurea & $\mathrm{U}$ \\
\hline *SEGAIBANA 40 SC & $\mathrm{H}$ & $\mathrm{U}$ & Yes & Nicosulfuron & Sulfonylurea & $\mathrm{U}$ \\
\hline $\begin{array}{l}\text { SUNHALOTHRINE } 2,5 \% \\
\text { EC }\end{array}$ & I & III & Yes & Lambda-cyhalothrin & Pyrethroid & II \\
\hline SUN-LAMBDA 2,5 EC & I & II & No & Lambda-cyhalothrin & Pyrethroid & II \\
\hline SUNPHOSATE 360 SL & $\mathrm{H}$ & II & Yes & Glyphosate & Phosphonoglycin & III \\
\hline SUPRAXONE & $\mathrm{H}$ & II & No & Paraquat & Bipyridylium & II \\
\hline \multirow{2}{*}{ THUNDER 45 O-TEQ } & \multirow{2}{*}{ I } & \multirow{2}{*}{ II } & \multirow{2}{*}{ Yes } & Imidacloprid & Neonicotinoid & II \\
\hline & & & & Beta-cyfluthrin & Pyrethroid & $\mathrm{Ib}$ \\
\hline
\end{tabular}




\begin{tabular}{|c|c|c|c|c|c|c|}
\hline Commercial formulation & $\begin{array}{l}\text { Type of } \\
\text { pesticide }\end{array}$ & $\begin{array}{l}\text { WHO } \\
\text { class }\end{array}$ & $\begin{array}{c}\text { CSP } \\
\text { registration } \\
\text { status }\end{array}$ & $\begin{array}{l}\text { Active substances } \\
\text { (a.s.) }\end{array}$ & Group of substances & $\begin{array}{l}\text { WHO } \\
\text { class of } \\
\text { a.s. }\end{array}$ \\
\hline \multirow{2}{*}{ *TIHAN175 O-TEQ } & \multirow{2}{*}{ I } & \multirow{2}{*}{ III } & \multirow{2}{*}{ Yes } & Spirotetremate & Tetramicacid & III \\
\hline & & & & Flubendiamide & Benzene-dicarboxamide & III \\
\hline TOPEXTRA 720 SL & $\mathrm{H}$ & II & Yes & 2,4 D-Amine salt & Aryloxyacide & II \\
\hline TRAZINE $500 \mathrm{SC}$ & $\mathrm{H}$ & II & No & Atrazine & Triazine & III \\
\hline
\end{tabular}

and Flubendiamide) and ACERO 84EC (Isoclast and Lambda-cyhalothrin) (11.5\%), EMAPYR (Emamectin benzoate, Pyriproxyfen) (2.9\%), LADABA (glyphosate) (1.4\%) and GLYPHADER (glyphosate) (0.7\%).

\subsection{WHO toxicity class}

The pesticides used by the producers are belongs to different classes of WHO toxicity, among identified pesticides 59.26\%, $22.22 \%$ and $18.52 \%$ are belongs to Class III, U and II respectively. In case of active substances, these are at $50.00 \%, 40 \%$ and $10 \%$ to class II, class III and class $U$ respectively.

\subsection{CMR toxicity and endocrine disruptors}

The pesticide found with producers contains 3 active substances (imidacloprid, paraquat and spirotetramate) with CMR effects. From the active substances that are not CMR, 29\% are possible carcinogens and $65 \%$ are possible reprotoxic, among which, thiram is both possible carcinogenic, reprotoxic and mutagenic. Deltamethrin and flubendiamide, also used in approved formulations, are endocrine disruptors. CMR and endocrine disruptors' information collected from Footprint PPDB are recorded in Table 4.

\section{Discussion}

\subsection{Surveys}

Around the RBMH, the pesticides are the subject of an unauthorized trade in general by the retailers, thus posing the problem of their control and distribution. A study conducted in 3 regions of western Burkina Faso also reported 97\% of
Table 4 Interpretation of CMR category and endocrine disruptor of the active substances of used pesticides (Footprint PPDB, 2018)

\begin{tabular}{|c|c|c|c|c|}
\hline Actives substances & Carcinogenicity & Mutagenicity & $\begin{array}{l}\text { Toxic for } \\
\text { reproduction }\end{array}$ & $\begin{array}{l}\text { Endocrine } \\
\text { disruption }\end{array}$ \\
\hline Chlorpyrifos-éthyl & No & No & No data & Possibly \\
\hline Deltamétrine & No & No & Possibly & Yes \\
\hline Diflufenican & No & No & Possibly & No data \\
\hline Diuron & Possibly & No & Possibly & Possibly \\
\hline Emamectine benzoate & No & No & Possibly & No data \\
\hline Flubendiamide & No & No & Possibly & Yes \\
\hline Flufenacet & No & No & Possibly & No data \\
\hline Glyphosate acide & Possibly & No & No & Possibly \\
\hline $\begin{array}{l}\text { Glyphosate } \\
\text { seld'isopropylamine }\end{array}$ & Possibly & No & No & No \\
\hline Haloxyfop-R-Méthyle & No & No data & No & No data \\
\hline Imidaclopride & No & Possibly & Yes & No data \\
\hline Indoxacarbe & No & No & Possibly & Possibly \\
\hline Isoclast (sulfoxaflor) & Possibly & No data & Possibly & No data \\
\hline Lambda-Cyhalothrine & No & No & Possibly & No \\
\hline Nicosulfuron & No & No & No data & No data \\
\hline Paraquat & Possibly & yes & Possibly & No \\
\hline Paraquat dichloride & No & No data & Possibly & No \\
\hline Pyriproxyfène & No & No & No & Possibly \\
\hline Spirotetramate & No & No & Yes & No \\
\hline Thirame & Possibly & Possibly & Possibly & Possibly \\
\hline
\end{tabular}


illegal retailers (Rotterdam Convention, 2016). Indeed, the supervision of the pesticides sale in Burkina Faso is governed under the Article 9 of the Law $\mathrm{N}^{\circ}$ 026-2017/AN on pesticides, this article stipulating that the activity must be authorized by an approval issued by the Ministry of Commerce (Assemblée Nationale, 2017). Also, low level of education of crop producers does not allow them to interpret the instructions of the packaging labels and meaning of pictograms (Fayomi et al., 1998; Ouédraogo et al., 2009; Belhadi et al., 2016). In addition, the long experience of pesticides use, age of producers and low level of PPE use, presage chronic risks. It is also evident that producers and retailers have close trading relationships related to the marketing and supply of pesticides (Khooharo et al., 2008). Further, Fan et al. (2015) recognize that in some cases, retailers are the only ones who provide information to producers but in maximum cases these retailers have low level of formal education. This type of relationship between illegal and less educated cotton producers and pesticide retailers, could lead to the distribution and use of pesticides harmful to health and environment. In addition, the major problem related to the production of cotton is the attack of insect pests that cause enormous damage on cotton. Thus, the choice of insecticides must base on products popularized by research. Factors such as poverty lead them to buy most of the credit products payable by deducting the gain from the sale of cotton at the end of the season (Sawadogo, 2016; Luna, 2019). In addition, a practice of associating the sale of pesticides with consumer products is also dangerous for health knowing that leakage of pesticides from packaging can occur and contaminate food products. This can be a source of intoxication for consumers.

Around the RBMH, surveys have shown that most of the producers use their ordinary country outfits, far from the standard of conventional PPE, adapted to the types and nature of pesticides. However, the use of PPE is recommended to prevent exposure and the risk of pesticide applicators becoming intoxicated (Hanshi, 2001; Khan et al., 2009; Norkaew et al., 2010; Yuantari et al., 2015). Cotton producers partly noted the unavailability, cost and discomfort of the combinations. The discomfort related to the wearing of the suits would partly be due to the climate which is warm during the campaign (Weng \& Black, 2015). Under this warm climatic condition, recommended clothes for pesticides spray might prevent the evaporation of sweating and affect different physiological mechanisms of central temperature regulation (Garrigou et al., 2008). This would create new hazards and health risk related to heat work. In addition, half of the producers reported cases of acute intoxication linked to certain pesticides including insecticides and glyphosate, confirming the same trend obtained during a study in western Burkina Faso in 2010 (Toé et al., 2013).

Some cotton producers mix pesticides before the treatments, which they believe will save time and efficiency. This practice should be banned because it might given the existence of possible health and environmental effects linked to chemical mixtures (Bull et al., 2006; Youness, 2013; Décosse, 2013). Use of empty packaging in incineration, abandonment in nature and domestic purpose was reported during the study, these findings are in agreement with the observation of Nonga et al. (2011); Gomgnimbou et al. (2009); Ouédraogo et al. (2009) and Devez (2004). The proper management of empty packaging is must and it should be regulated by law.

Observation of the reentry time is crucial to avoid the risk of posttreatment intoxication. In Burkina Faso, field work involves several age groups and both sexes, among these, children and women are more sensitive to the effects of pesticides. Few producers unfortunately observe a post-treatment safety delay of at least 48 hours, the minimum time often recommended when using moderately hazardous pesticides (Samuel, 2018).

The presence of several water points in the fields or nearby and their use for the preparation of dilutions during treatment, might became an important health issue for human and animal those who consumed water of these water points. Some time, waters points are exposed to a potential contamination from the drift of treatment, runoff or infiltration. Further, different sources of pesticide might become potential sources of pollution of the water table through the water flows (Soleri, 2013).

From the recommendations and suggestions made, the producers showed that they are probably aware of the risk associated with pesticides and the means for a secure management. Indeed, the reduction in the number of pesticide treatments, the accessibility of medical visits after treatment, the availability and accessibility of PPE indicated the level of awareness of the danger posed by pesticides and the need to preserve them. Thus, the use of pesticides under the conditions described for the producers appears as a necessary evil which they now seem unable to cope with.

\subsection{Characteristics of pesticides and risks to health}

Apart from the glyphosate product KALACH, all the pesticides (herbicides, insecticides and fungicides) used by the producers have been identified at the level of the retailers. This suggests the global consideration of producers' needs by retailers. Thus, from a pesticide risk management perspective, there is a need to rigorously regulate the distribution of pesticides to avoid the use of unauthorized pesticides. For example, Beta-Cyfluthrin, class Ib, which is reported only from retailers and provided to producers by SOFITEX the previous campaign, is a prohibited pesticide (UTZ, 2015) that should be subject to a restriction of use in the context of agricultural practices in Burkina Faso.

Insecticides PYRINEX QUICK 424EC (deltamethrin and chlorpyrifos-ethyl), TIHAN 175 O-TEQ (spirotetramate and 
flubendiamide), ACERO 84EC (isoclast, lambda-cyhalothrin), EMAPYR (emamectin benzoate, pyriproxyfen), and two glyphosate herbicides (LADABA, GLYPHADER) have been related to acute intoxication. The insecticides are quantitatively the most used, in three periods of treatment and repeated twice each by campaign (Traoré, 2008). These are more toxic to humans in terms of targets and mechanisms of action. These pesticides are composed of spirotetramate (Footprint PPDB, 2018), deltamethrin, emamectin benzoate, lambda-cyhalothrin, and isoclast, which are likely to be carcinogens or reprotoxic (Footprint PPDB, 2018), which may lead to chronic poisoning risks in case of repeated use. Glyphosate remains a highly controversial herbicide molecule classified as a possible carcinogen (Footprint PPDB, 2018) and is the most widely used herbicide molecule encountered. In addition, the pesticides used are mainly Class III (slightly dangerous) but contain CMR active substances or endocrine disruptors are available in these (Footprint PPDB, 2018). Also, paraquat based formulations are banned in Burkina Faso (FAO, 2018). Results of current study are in agreement with the findings of previous researchers (Toé et al., 2013; Convention de Rotterdam, 2016).

\section{Conclusion}

The study of pesticide characterization and analysis of the practices of pesticide stakeholders in rural areas made possible to understand the types of used herbicides or pesticides and risk associated with the use of these two. The harmful agricultural practices and dangerousness / toxicity of the used molecules lead to the exposure of intoxication in producers. Poor conditions involved the distribution of toxic and unapproved pesticides (CMR, endocrine disruptors, ecotoxicants, etc.), use of inappropriate PPE, short re-entry time, mixing of products, etc. Also, the non-formal marketing of pesticides and the illiteracy of the producers constitute important risk factors. Further, studies evaluating human exposure and environmental pollution are expected to better characterize the risk associated with pesticides in and around the Bala RBMH.

\section{Conflict of Interest}

The authors declare that there is no conflict of interest.

\section{References}

Achaleke J, Brévault T (2010) Inheritance and stability of pyrethroid resistance in the cotton bollworm Helicoverpa armigera (Lepidoptera: Noctuidae) in Central Africa. Pest Management Science 66:137-141. doi: 10.1002/ps.1843.

Assemblée Nationale (2017) LOI Nº26-2017/AN portant contrôle de la gestion des pesticides au Burkina Faso, Pp. 29.
Badiane D, Gueye MT, Coly EV, Faye O (2015) Gestion intégrée des principaux ravageurs du cotonnier au Sénégal et en Afrique occidentale. International Journal of Biological Chemical Sciences 9: 2654-2667.

Belem M (2008) Les galeries forestières de la Réserve de la Biosphère de la Mare aux Hippopotames du Burkina Faso: caractéristiques, dynamique et ethnobotanique, Thèse de doctorat d'Etat,Université de Ouagadougou, Pp. 208.

Belhadi A, Mehenni M, Reguieg L, Yakhlef H (2016) Pratiques phytosanitaires des serristes maraîchers de trois localités de l'Est des Ziban et leur impact potentiel sur la santé humaine et l'environnement. Revue Agriculture 1(S): 09-16.

Bull S, Fletcher K, Boobis AR, Battershill JM (2006) Evidence for genotoxicity of pesticides in pesticide applicators: a review. Mutagenesis 21: 93-103.

Calvet R, Barriuso E, Bénoit P, Charnay MP, Coquet Y (2005) Les pesticides dans le sol : conséquences agronomiques et environnementales. Éditions, France Agricole.

Convention de Rotterdam (2016) Utilisation Des Pesticides Agricoles Dans Trois Régions À L'ouest Du Burkina Faso Et Evaluation De Leur Impact Sur La Sante Et L'environnement : cas des Régions de la Boucle du Mouhoun, des Cascades et des HautsBassins. Rapport d'étude réalisé par l'institut de recherche en science de la santé, direction régionale de l'ouest (IRSS/DRO)/ Centre National de la Recherche Scientifique et Technologique (CNRST), Burkina Faso, Pp. 100.

Décosse F (2013) «Entre «usage contrôlé », invisibilisation et externalisation. Le précariat étranger face au risque chimique en agriculture intensive. Sociologie du travail 55: 322-340.

Devez A (2004) Caractérisation des risques induits par les activités agricoles sur les écosystèmes aquatiques. Thèse de doctorat : École Nationale du Génie Rural des Eaux et des Forêts (ENGREF), HydroSciences Montpellier (HSM).

Fan L, Yang HNX, Qin W, Bento CPM, Ritsema CJ, Geissen V (2015) Factors affecting farmers' behaviour in pesticide use: Insights from a field study in northern China. Science of the Total Environment 537: 360-368.

FAO (2015) Measuring Sustainability in Cotton Farming Systems. Towards a Guidance Framework. Report prepared by the ICAC Expert Panel on Social, Environmental and Economic Performance of Cotton Production with the FAO Plant Production and Protection Division. ISBN 978-92-5-208614-7. Rome, Italie, available on http://www.fao.org/3/a-i4170e.pdf. 
FAO (2018) Mise en œuvre de la convention de Rotterdam à travers une collaboration régionale en Afrique de l'ouest: l'exemple des pays du comité permanent inter-Etats de lutte contre la sécheresse dans le sahel (CILSS), Pp. 24.

FAO/OMS (2014) Code de conduite international sur la gestion des pesticides. ISBN 978-92-5-208548-5. Rome, Italie, Pp. 58.

Fayomi B, Lafia E, Fourn L, Akpona S, Zohoun T (1998) Connaissance et comportement des utilisateurs de pesticides au Bénin. African Newsletter 2: 40-43.

Footprint PPDB (2018) The Footprint Pesticide Properties Data Base. Available on https://sitem.herts.ac.uk/aeru/iupac/atoz.htm accessed on November 30, 2018.

Garrigou A, Baldi I, Dubuc P (2008) Apports de l'ergotoxicologie à l'évaluation de l'efficacité réelle des EPI devant protéger du risque phytosanitaire : de l'analyse de la contamination au processus collectif d'alerte. Perspectives interdisciplinaires sur le travail et la santé, Pp. 21.

Gomgnimbou APK, Savadogo PW, Nianogo AJ, MillogoRasolodimby J (2009) Usage des intrants chimiques dans un agrosystème tropical : diagnostic du risque de pollution environnementale dans la région cotonnière de l'est du Burkina Faso. Biotechnology, Agronomy, Society and Environment 13 499-507.

Hanshi JA (2001) Use of pesticides and personal protective equipment by applicators in a Kenyan district. African News letter on Occupational Health and Safety 11, number 3: 74-76.

Khan DA, Shabbir S, Majid M, Ahad K, Naqvi TA, Khan FA (2009) Risk assessment of pesticide exposure on health of Pakistani tobacco farmers. Journal of Exposure Science and Environmental Epidemiology.DOI: 10.1038/jes.2009.55.

Khooharo AA, Memon RA, Mallah MU (2008) An empirical analysis of pesticide marketing in Pakistan. Pakistan Economic and Social Review 46: 57-74.

Luna JK (2019) The chain of exploitation: intersectional inequalities, capital accumulation, and resistance in Burkina Faso's cotton sector. The Journal of Peasant Studies 46: 1413-1434, DOI 10.1080/03066150.2018.1499623. 23p.

MECV (2011) Analyse économique du secteur du coton, liens pauvreté et environnement. Burkina Faso, Ministère de l'Environnement et du Cadre de Vie (MECV). Projet Initiative PauvretéEnvironnement (IPE), Rapport final, août 2011, 60p.

Nonga HE, Mdegela RH, Lie E, Sandvik M, Skaare JU (2011) Assessment of farming practices and uses of agrochemicals in
Lake Manyara basin. Tanzania African Journal of Agricultural Research 6: 2216-2230.

Norkaew S, Siriwong W, Siripattanakul S, Robson M (2010) Knowledge, attitude, and practice (kap) of using personal protective equipment (PPE) for chilli growing farmers in huarua sub-distfuct, mueang district, ubonrachathani province, Thailand. Journal of Health Research 24: 93- 100.

Ouédraogo M, Tankoano A, Ouédraogo ZT, Guissou IP (2009) Etude des facteurs de risques d'intoxications chez les utilisateurs de pesticides dans la région cotonnière de Fada N'Gourma au Burkina Faso. Environnement, Risques \& Santé 8: 343-347.

Ouédraogo M, Toé AM, Ouédraogo TZ, Guissou PI (2011) Pesticides in Burkina Faso: Overview of the Situation in a Sahelian African Country, Pesticides in the Modern World -Pesticides Use and Management. Intech Open, DOI: 10.5772/16507. pp 36-48.

PAN UK (2018) Pesticide Concerns in Cotton. Available at https://www.pan-uk.org/cotton/ accessed on July 21, 2019.

Rea ML, Parker AR (2014) Designing and Conducting Survey Research A Comprehensive Guide, Fourth Edition. Available on https://eva.udelar.edu.uy/pluginfile.php/876387/mod_resource/cont ent/0/Louis\%20M.\%20Rea\%2C\%20Richard\%20A.\%20Parker-

Designing\%20and\%20Conducting\%20Survey\%20Research_\%20

A\%20Comprehensive\%20Guide-Jossey-

Bass\%20\%282014\%29.pdf. Accessed on June 15, 2018.

Rotterdam Convention (2016) Rotterdam Convention Website and Stockholm Convention Website: http://www.pic.int/Countries/ Statusofratifications/tabid/1072/language/en-US/Default.aspx. http://chm.pops.int/Countries/StatusofRatifications/PartiesandSign atoires/tabid/4500/Default.aspxs accessed on July 21, 2019.

Samuel O (2018) Proposition et validation de critères de détermination de délais de réentrée pour les pesticides utilisés en pépinières forestières. Centre de toxicologie du Québec. Bulletin d'information toxicologique 12: 2 .

Saravi SSS, Shokrzadeh M (2011) Role of Pesticides in Human Life in the Modern Age: A Review, Pesticides in the Modern World - Risks and Benefits. Intech Open DOI: 10.5772/18827. 12p. Available at https://www.intechopen.com/books/pesticides-inthe-modern-world-risks-and-benefits/role-of-pesticides-in-humanlife-in-the-modern-age-a-review access on June 15, 2018.

Sawadogo MFWE (2016) Biotechnology Cotton in Burkina Faso: Trajectory of an Innovation in a Development Context. ISTE OpenScience - Published by ISTE Ltd. London, UK, Pp. 15. 
Soleri R (2013) Etude de la pression phytosanitaire exercée sur différents lacs du Burkina Faso par méthode d'échantillonnage passif. Mémoire de stage de Master $2^{\text {ème }}$ année Mention : Contaminants Eau Santé, Université de Montpellier 2, sciences et techniques, France. 50 p.

Son D, Somda I, Legreve A, Schiffers B (2017) Pratiques phytosanitaires des producteurs de tomates du Burkina Faso et risques pour la santé et l'environnement. Cahiers Agricultures 26 250-255.

Sory S (2011) Etude du marché des herbicides dans la production du riz: cas des sitesde Bagré, Barna, Bazon et Niena-Dionkélé. Mémoire d'Ingénieur, IDR/UPB, BoboDiou1asso, Burkina Faso, $63 p+a n n e x e s$.

Toé MA, Ouédraogo M, Ouédraogo R, Ilboudo S, Guissou IP (2013) Pilot study on agricultural pesticide poisoning in Burkina Faso. Interdisciplinary Toxicology 6: 185-191.

Traoré O (2008) Les succès de la lutte intégrée contre les ravageurs du cotonnier en Afrique de l'Ouest. Communication à la $67^{\text {ème }}$ réunion plénière de l'ICAC. Ouagadougou (Burkina Faso), 16-21 November 2008, Pp.11.
UTZ (2015) Liste des pesticides prohibés et liste des pesticides sous surveillance, Version 1.0. 19p.

Weng CY, Black C (2015) Taiwanese farm workers' pesticide knowledge, attitudes, behaviors and clothing practices. International Journal of Environmental Health Research 25: 685-696.

WHO (2010) The WHO Recommended Classification of Pesticides by Hazard and Guidelines to Classification 2009. 78p. available

at https://apps.who.int/iris/bitstream/handle/10665/44271/978924154 7963_eng.pdf?sequence $=1 \&$ isAllowed $=y$ accessed on November 13, 2019.

Youness M (2013) Impact de la formulation et du mélange de deux pesticides (mésotrione et tébuconazole) sur leur biodégradation et la croissance de microorganismes. Université Blaise Pascal Clermont-Ferrand II, 2013. Français, Pp. 289.

Yuantari GCM, Van Gestel AMC, Van Straalen MN, Widianarko B, Sunoko RH, Shobib NM (2015) Knowledge, attitude, and practice of Indonesian farmers regarding the use of personal protective equipment against pesticide exposure. Environmental Monitoring and Assessment187:142. 